\title{
A self-organizing quasi-linear ARX RBFN model for nonlinear dynamical systems identification
}

\begin{abstract}
The quasi-linear ARX radial basis function network (RBFN) model has shown good approximation ability and usefulness in nonlinear system identification and control. It has an easy-to-use structure, good generalization and strong tolerance to input noise. In this paper, we propose a self-organizing quasi-linear ARX RBFN (QARX-RBFN) model by introducing a self-organizing scheme to the quasi-linear ARX RBFN model. Based on the active firing rate and the mutual information of RBF nodes, the RBF nodes in the quasi-linear ARX RBFN model can be added or removed, so as to automatically optimize the structure of the quasilinear ARX RBFN model for a given system. This significantly improves the performance of the model. Numerical simulations on both identification and control of nonlinear dynamical system confirm the effectiveness of the proposed self-organizing QARX-RBFN model.
\end{abstract}

Keyword: Nonlinear dynamical system; System identification and control; Quasi-linear ARX model; Self-organization; Radial basis function network 